\title{
COVID-19 and Nervous System: Under Estimated Clinical and Prognostic Aspects
}

\author{
Alyne Barreto Mesquita de Goés ${ }^{1}$, Bruno Barreira Cardoso ${ }^{1}$, Francisco de Assis Fernandes \\ Tavares $^{1}$, Rebecca Renata Lapenda do Monte ${ }^{1}$, Renata Carneiro Melo ${ }^{1}$, Irami Araújo- \\ Neto $^{1}$, Renato Serquiz Elias Pinheiro ${ }^{2}$, Amália Cinthia Meneses Rêgo ${ }^{3,4}$ and Irami Araújo- \\ Filho $^{5,6,7^{*}}$
}

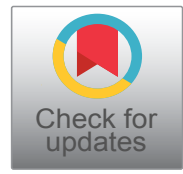

${ }^{1}$ Undergraduate Student of Medicine at UnP, Potiguar University, Laureate International Universities, Natal/Brazil ${ }^{2}$ Neurologist and Professor at Potiguar University/UnP, Laureate International Universities, Natal/Brazil ${ }^{3}$ Postgraduate Program in Biotechnology at Potiguar University/UnP, Laureate International Universities, Natal/Brazil ${ }^{4}$ Teaching and Research Manager, School of Health, League Against Cancer Natal, RN/Brazil ${ }^{5}$ Postgraduate Program in Biotechnology at Potiguar University/UnP, Laureate International Universities, RN/Brazil ${ }^{6}$ Full Professor Department of Surgery, Federal University of Rio Grande do Norte, Brazil

${ }^{7}$ Full Professor, Department of Surgery, Potiguar University, Brazil

*Corresponding author: Irami Araújo-Filho, MD, Ph.D, Postgraduate Program in Biotechnology at Potiguar University/ UnP, Laureate International Universities; Full Professor, Department of Surgery, Federal University of Rio Grande do Norte; Full Professor, Department of Surgery, Potiguar University, Brazil, Tel: +55-84-98876-0206, Fax: +55-84-3342-5079

\begin{abstract}
The coronavirus pandemic transformed the world abruptly due to the speed of transmission and high morbidity and mortality. Many deaths have been quantified, and the scientific community intensifies the search for molecular targets, protein sequences and polymorphismson SARS-CoV-2, to improve the clinical evolution and survival of patients. Initially, COVID-19 was described with respiratory changes, flu, and fever. With the spread of the disease, clinical manifestations were observed in other organ systems, still unknown. In this sense, the present study describes the main neurological changes and laboratory findings. The literature review was identified in the central databases: Scielo, Google Scholar, PubMed/MedLine, Embase, and Cochrane Database. Twenty-five articles related to the theme were chosen, including reviews, case series, cohort, and retrospective studies. Neurological manifestations were predominantly anosmia/hyposmia, dysgeusia, ataxia, and seizures. According to the latest published studies, attention must be paid to isolated initial neurological events.
\end{abstract}

\section{Keywords}

COVID-19 pandemic, SARS-CoV-2 infection, Coronavirus, Central Nervous System Viral Diseases, Peripheral Nervous System Diseases, Injury

\section{Introduction}

The new coronavirus appeared in the city of Wuhan in China, and, due to its high infectivity, it spread quickly to the whole world. SARS-CoV-2 was responsible for the critical state of the pandemic that we are experiencing today. The clinical syndrome caused by SARS-CoV-2 was called COVID-19 [1,2].

The signs and symptoms are clinically in distinguishable from those of the flu. Studies have described fever (43.8\% on admission and $88.7 \%$ during hospitalization), cough $(67.8 \%)$, fatigue $(50 \%)$, headache $(8 \%)$, and diarrhea (5\%). However, the disease affects other organ systems and evolves with varied clinical manifestations $[1,3]$.

In addition to changes in the respiratory system, patients with COVID-19 may have neurological syndromes, loss of involuntary breathing process, impairment of the brainstem, ataxia, loss of smell, seizures, among others. These manifestations are usually alarming and signal a worse prognosis [1].

Citation: de Goés ABM, Cardoso BB, Tavares FAF, do Monte RRL, Melo RC, et al. (2020) COVID-19 and Nervous System: Under Estimated Clinical and Prognostic Aspects. Int J Neurol Neurother 7:100. doi. org/10.23937/2378-3001/1410100

Received: May 26, 2020: Accepted: June 22, 2020: Published: June 24, 2020

Copyright: (c) 2020 de Goés ABM, et al. This is an open-access article distributed under the terms of the Creative Commons Attribution License, which permits unrestricted use, distribution, and reproduction in any medium, provided the original author and source are credited. 
Neurological changes seen in patients with severe infection include acute cerebrovascular events, impaired consciousness, areflexia, paraesthesia, and muscle injury [3].

As the emergence of the pandemic, the recognition of neurological clinical findings is in its early stages. Thus, little is known about the syndromic complexity of COVID-19. Still, it is believed that the neurological disorders of the disease are similar to the data previously described by the infection of SARS-CoV-1, a virus of the same family [3].

No vaccine or antiviral treatment specific to COVID-19 has yet been shown to be effective. For this reason, several preventive measures have been suggested to minimize the damage caused by the disease, personal protection, social distance, adequate hygiene measures, and isolation of confirmed cases. Identifying and treating patients as quickly as possible, especially patients considered to beat high risk, is crucial to reducing the mortality rate [4].

Mortality is higher among elderly patients and patients with comorbidities, including diabetes, cardiac, respiratory disorders, and immunosuppressed [4].

This article describes the main neurological signs and symptoms reported in the literature and correlates these findings with etiopathogenic mechanisms, laboratory tests, and prognostic factors.

\section{Methods}

The present study was carried out based on searches in PubMed/Medline, Scopus, Scielo, Embase, and Web of Science databases in addition to the Google Scholar search, considered as a source of gray literature, as it does not contain peer-reviewed articles. Studies related to neurological manifestations of the coronavirus were selected, being chosen through the combination of the following keywords: neurological signs and COVID-19. All relevant studies published between 2019 and 2020 were included in the cohort, systematic review, case-control, cross-sectional, case series studies, and randomized clinical trials. The filters chosen were studies carried out in humans, complete articles, and recently published, totaling twenty-five articles analysis, review, and selection of the articles were made by peers blindly, from reading the title and summary of the study, with a third reviewer in case of disagreement between the other two reviewers.

\section{Results and Discussion}

The coronavirus family comprises viruses that cause diseases with different clinical manifestations, which can vary from a common cold to severe clinical conditions. SARS-CoV-2 is the new corona virus, first detected in China and spreading globally [1].

The average age of onset is $35-55$ years of age, with a lower prevalence in children. Most patients are male, and the main risk groups are immunosuppressed patients with renal and hepatic dysfunction. Transmission can occur through droplets, aerosols, and contaminated surfaces, when in contact with the mouth, nose, or eyes of susceptible individuals. There is an increase in the number of cases transmitted through the feces of infected patients [5].

To date, there is no specific consolidated treatment regime for COVID-19, which is why the importance of symptomatic management is essential. Thus, a typical symptoms must be taken into account during anamnesis and physical examination of patients, since the evolution of the disease, in these cases, canbe more severe. Patients may experience respiratory, digestive, liver problems, and disorders of the nervous system [6-8].

Nath $A$, et al. Subdivided these into manifestations that occur at the time of infection and post-infection events. Encephalopathy, hypoventilation, viral meningitis, anosmia, ageusia, encephalitis, stroke, myositis, and acute hemorrhagic encephalopathy are parainfectious symptoms. Transverse myelitis, Guillan-Barré syndrome, sensory neuropathy, brainstem encephalitis, and acute transverse encephalomyelitis, usually occur after COVID-19 infection [7-9].

\section{Health system}

The measurement of neurological disorders based on signs and symptoms, as well as the patients' prognosis, can be assessed through the protocol developed by the World Health Organization (WHO) in February 2020. In this, respiratory findings are addressed; however there is a specific column to detect the neurological aspects involved $[8,9]$.

It is important to note that these clinical presentations are being released to the world in real-time by the number of "First Few $X$ cases (FFX) and their close contacts" by WHO. Several cases have been described, with nonspecific manifestations such as loss of smell, headache, seizures, and hemiparesis $[8,10]$.

\section{Pathophysiology}

SARS-COV and MERS-COV have neuro-invasive properties, which can be detected in the human brain [9]. The pathophysiology of the lesions has not yet been clarified concerning COVID-19; however, it is believed that it occurs via hematogenous, reaching the Central Nervous System (CNS) or by retrograde neuronal dissemination, due to the proximity of the cribriform plate of the ethmoidal bone. Depending on this theory, there is a report of hyposmia in several patients [11].

Also, it is known that the primary target cells for SARS-CoV-2 are the epithelial cells of the respiratory and gastrointestinal tract, which contain the angiotensin-converting enzyme 2 (ACE2), which is also present in the CNS and skeletal musculature. This enzyme is usual- 
ly used by the virus to invade cells, for which it presents tropism [2,10-12].

Inhalation of SARS-CoV-110 or MERS-CoV-11 resulted in the rapid invasion of viral particles in the brain, possibly through the olfactory bulb via the trans-synapticroute. Several viruses penetrate this pathway into the peripheral nerves and spread through the CNS through synaptic contacts [5-7].

The brainstem, where the neuronal respiratory bulb is located in the medulla, has been severely infected by both types of viruses, which can contribute to respiratory failure. On the otherhand, it was observed that when nasal viral loads were inhaled in low concentrations, only the CNS was colonized, with the absence of viruses in other tissues, including the lungs, corroborating the existence of the neurotropism of the coronavirus strains by the CNS [2-4].

Due to retrograde neuronal dissemination, $\mathrm{Li}$, et al. Advocate that the use of protective masks and antiviral drugs at the beginning of the disease should be encouraged to prevent CNS involvement [11-13].

A severe complication of COVID-19 infection is Acute Disseminated Encephalomyelitis, a syndrome described in patients with $\mathrm{HCOV}-\mathrm{OC} 43$, which occurs approximately two weeks after infection. This syndrome is also expected to happen in SARS-CoV-2 [1,7].

The presence of the virus in the brain stem can affect chemosensory neural cells associated with respiratory and cardiovascular regulation, as well as neurons in the bulbar respiratory center (brainstem), affecting ventilatory lung function. An additional factor to the hypothesis that the nasal route may contribute to the entry of the virus in the brain is provided by clinical observations of anosmia, a new and profound mark in individuals infected with SARS-CoV-2 [1-3].

The infection by SARS-CoV-2 in the CNS triggers an intense systemic inflammatory reaction with a massive release of cytokines, chemokines, and other inflammatory mediators, with subsequent breakdown of the blood-brain barrier and amplifies the viral neuro-infection process [14-16].

The higher the plasma concentration of cytokines, the greater the need for intensive care. Pre-clinical and clinical studies have shown that systemic inflammation promotes neuroinflammation that severely affects cerebral homeostasis and causes the death of central and peripheral neurons $[2,11,12]$.

\section{Neurological symptoms}

According to Baig, et al., COVID-19 and SARS-CoV-1 have similar taxonomy. SARS-CoV-1 promotes neurological deficits and has already been isolated in brain tissue. In this sense, the coronavirus is expected to follow the same trend [3].
Neurological manifestations are classified into two categories: Those that affect the Central Nervous System (CNS) and those that affect the Peripheral Nervous System (PNS). Clinical findings related to the CNS include vertigo, headache, impaired consciousness, acute vascular brain disease, ataxia, and epilepsy. SNP involvement causes hyposmia, neuralgia, and hypogeusia $[13,17]$.

As for SARS-CoV-2, there is no exact description of what signs and symptoms to investigate or which neurological tests are indispensable in the physical examination of patients, as some, such as hyposmia and hypogeusia, are usually so mild that they are not present in most of the cases $[18,19]$.

However, if there is a report or the presence of deficits, it is essential to perform laboratory tests, such as urea, creatinine, electrolytes, and blood gas analysis $\left(\mathrm{PO}_{2}\right.$ and $\left.\mathrm{PCO}_{2}\right)$, as the results can corroborate for a possible primary or secondary involvement of the CNS $[1,3]$.

The American Academy of Otorhinolaryngological Surgery (AACO) recently released that patients with COVID-19 may experience dysgeusia or hyposmia. These are the primary evidence that should be researched. It is believed that the virus causes inflammation in the olfactory nerves, instead of damaging the structure of the receptors, as the olfactory system can always fight pathogens and their dysfunctions are temporary. Generally, these same patients experience loss of taste $[3,14]$.

Based on the cases reported by AACO, it is recommended to investigate COVID-19 if the patient says anosmia, hyposmia, or dysgeusia in the absence of other respiratory diseases, such as acute or chronic rhinosinusitis and allergic rhinitis. The "COVID-19 Anosmia Report Tool for Clinicians" was made available by AAOHNS to facilitate this diagnosis $[14,15]$.

Anosmia is mostly transient, but it has been seen that in some patients, it has become permanent. As the lesion occurs due to invasion of the olfactory pathways, the monitoring of these patients should be encouraged for an extended period, to ensure that other neuroinflammatory diseases can be ruled out $[7,20]$.

The meningitis clinic is controversial, as some studies report that neuronal deaths with inflammation of the brain substance have not been detected. However, Wu, et al. report a case from Hospital Beijing Ditan, identified employing the genomic sequencing technique of the presence of SARS-CoV-2 in the cerebrospinal fluid. The patient was diagnosed with viral encephalitis, and SARS-CoV-2 attacked the central nervous system. The virus has mechanisms of direct invasion to the nervous system, in addition to the immune response to the pathogen $[3,9,10]$.

It is inquired about the acute toxic viral encephalitis syndrome, from manifestations such as headache, con- 
sciousness disorder, and cerebral edema seen in autopsies. Thus, it is necessary to analyze the CSF of patients with these manifestations in search of the viral genome and inflammatory cells $[4-6,10]$.

In Japan and China, two cases of meningoencephalitis and encephalitis have been reported, respectively. Both had generalized seizures and acute respiratory distress syndrome. One of them presented lesions in the temporal lobe with adjacent ventriculitis and injuries in the paranasal sinuses [15-17].

The exciting thing was that the virus could be isolated in the CSF, but it was not detected in the nasal swabs. However, it is essential to emphasize that CSF should only be collected in cases where neuroimaging tests do not clarify the patient's symptoms or in cases of infectious or autoimmune processes $[7,18]$.

In a series of cases with 214 patients, neurological symptoms were observed in $36.4 \%$, being more common in patients with severe respiratory status (45.5\%), with reports of acute cerebrovascular events, impaired consciousness, and muscle injury $[1,3]$.

In patients described with severe infection, $5.7 \%$ developed cerebrovascular diseases, such as stroke. Evidence indicates that SARS-CoV-2 amplifies the coagulation cascade, resulting in a state of hypercoagulability that causes arterial and venous occlusions in the brain. Besides, the acute inflammatory response syndrome and the previous risk factors of the patients favor the onset of stroke $[1,10,19]$.

A stroke occurs in patients without risk factors. The explanation for this is that the ACE2 viral receptor is present in endothelial cells, including the cerebral vascular system [7]. The relationship between viral infection and cerebral hemorrhage remains to be investigated. The connection of the virus with the angiotensin-converting enzyme 2 (ACE2) may be involved in brain hemorrhages, especially in hypertensive individuals [11-13,20].

After SARS-CoV-2 infection, the expression and function of ACE2 proteins are reduced. As the expression of ACE2 in hypertensive patients is low, SARS-CoV-2 disease is more likely to induce cerebral hemorrhage in these patients, since the ability to lower blood pressure will be reduced [14,21].

A second hypothesis highlights that patients with COVID-19 often suffer from coagulopathy and prolonged prothrombin time, factors that can contribute to secondary cerebral hemorrhage $[15,22]$.

COVID-19 raises D-dimer levels, which is related to thrombotic vascular events. Previous studies have reported cases of secondary cerebral infarction in SARS. Thus, COVID-19 has the potential to induce venous or arterial infarctions in the brain [5-7,15].

Acute Necrotizing Encephalopathy (ENA) is a rare complication of influenza and other viral infections, re- lated to intracranial cytokine storms. Accumulated evidence suggests that some subgroups of patients with severe COVID-19 may experience this storm of cytokines, affecting the neurological system, just as it does in the lungs $[7,16]$.

Poyiadji, et al. Reported the first case of ENA associated with COVID-19. A 50-year-old airline employee had a 3-day history of cough, fever, and altered mental status. The initial laboratory examination was negative for influenza, and a nasopharyngeal swab sample confirmed the diagnosis of COVID-19.

However, the CSF bacterial culture did not show growth after three days, and tests for herpes simplex virus 1 and 2, varicella-zoster virus, and West Nile virus were negative. It was not possible to test the presence of SARS-CoV-2 in the CSF. Computed tomography (CT) images of the skull without contrast showed symmetrical hypoattenuation within the bilateral medial thalamus with angiogram and normal CT venogram. Cerebral magnetic resonance images showed lesions that increase the hemorrhagic border in the bilateral thalamus, medial temporal lobes, and subinsular regions. Intravenous immunoglobulin was administered, and steroids were not started due to the risk of respiratory impairment [16].

In the literature, there is a description of seizures, and the definition of these cases is worrisome, as it is essential to differentiate with febrile seizures, observed in patients with high fever due to COVID-19 [3,8].

The first description of COVID-19 associated with seizures occurred in a 30-year-old woman admitted to the Neurological Emergency Room at Bou Ali Sina Hospital, Mazandaran Province, Iran. The patient reported that she had had a drycough for five days, followed by fever and fatigue, presenting generalized tonic-clonic seizures, for the first time, two days before admission. There was no family history of convulsive disorders or previous crises. Lumbar puncture was performed, and the cerebrospinal fluid (CSF) showed healthy proteins and glucose, with a count of 5 lymphocytes, without bacterial growth. Chest tomography showed focal ground-glass opacities, and nasal and pharyngeal samples were positive for COVID-19 [17].

The causes of seizures are due to primary infection or reactivation of the latent virus. There are several pathophysiological mechanisms of seizures in patients suffering from viral infections, including direct infiltration of brain tissue, production of toxins by the virus, or production of inflammatory mediators by the brain. Concerning coronavirus, the seizure occurs due to encephalitis, virus invasion to the brain, or by the toxic effect of inflammatory cytokines, which can lead to neuronal hyperexcitability via activation of glutamate receptors [17].

Neurological impairment is prevalent at the onset of 
the disease, with a median of 2 days. In the same series of cases, patients demonstrated hemiparesis and then evolved with respiratory changes. Others vaguely revealed themselves with fever and headache [1].

Syncope was another manifestation mentioned. The exciting thing, in this case, was the absence of other respiratory symptoms and imaging exams without changes. Thus, the treatment was delayed for hours, which resulted in the exposure of other patients without isolation.

In the current situation of a pandemic, simple radiography of a normal chest and atypical signs of infection, such as syncope, should not rule out COVID-19. For this reason, we emphasize the importance of knowing the clinical manifestations arising from the involvement of organs and systems other than the respiratory [18].

There are few reports of transverse myelitis. After presenting symptoms suggestive of COVID-19, one case was described in China, in which the patient developed acute flaccid paralysis with upper extremity paresis, lower extremity plegia, and hyporeflexia, in addition to intestinal and bladder incontinence. This patient was treated with ganciclovir, lopinavir/ritonavir, moxifloxacin, meropenem, glutathione, dexamethasone $(10 \mathrm{mg} /$ day/10 days), immunoglobulin ( $15 \mathrm{~g} /$ day/7 days) and vitamin B12. There was a slight improvement at the time of hospital discharge [7].

In the literature, there are reports of sensory changes, presence of post-infectious sensory neuropathy with paresthesias, pain, and upward progression in some patients considered a variant of Guillain-Barré syndrome [7].

Recently, a report of a 61-year-old woman was published who presented acute symmetrical weakness in the lower limbs and generalized fatigue, evolving with tendinous areflexia in the legs and feet and loss of strength in the upper arms. Laboratory tests at admission showed important lymphocytopenia and thrombocytopenia. However, there were no complaints of fever, cough, dyspnea, diarrhea, or precordial pain. The patient underwent a nerve conduction study, which showed the presence of demyelinating neuropathy, suggestive of Guillain-Barré syndrome [19]. On the eighth day of evolution, the patient developed a drycough and a high fever, leading the medical team to request chest CT and oropharyngeal RT-PCR, with positive results for COVID-19 [19].

Since then, Guillain-Barré syndrome has come to be considered a para-infectious affection, instead of the classic post-infectious profile of the syndrome. It is essential to mention that there were no cases of SARSCoV-2 infection in the neurological ward, where the patient was, nor the presence of the disease in close contacts $[16,19]$.

The necessary evaluation of more epidemiological data in the clinical investigation of patients with suspected COVID-19, symptoms such as those that the patient had at the beginning of the picture should be taken into account as atypical manifestations of the disease [19].

The identification of patients with a confirmed diagnosis of COVID-19 who have neurological symptoms or not essential, as detecting specific changes, such as hypoxia, respiratory or metabolic acidosis, can alter the course of the disease. This approach is necessary for targeted and early treatment of these patients since CNS involvement requires more specific care and has a worse prognosis $[1,3]$.

Besides, there is a strong association between systemic inflammation and depressive syndromes. Certain infections, such as the flu, can increase the risk of depressive episodes in approximately $60 \%$ of cases. Thus, it was seen that the severe respiratory failure that accompanies COVID-19 triggers lasting hypoxia, which affects the brain and causes cognitive changes.

Finally, the neuroinflammatory process associated with functional brain damage explains the clinical experience that, even in patients who have overcome severe pneumonia, the onset or progression of cognitive impairment related to behavioral changes is observed.

Delirium, cognitive deficits, and behavioral abnormalities are related to systemic inflammation related to conditions of prolonged hypoxia, persistent and uncontrolled neural inflammation - responsible for damage to the hippocampus and cortical areas associated with cognitive functions and behavioral changes [2].

Upto the time of publication of this study, to our knowledge, there was a report of two patients infected with SARS-CoV-2 who had symptoms of Miller Fisher's syndrome (SMF) and cranial polyneuritis. SMF consists of a variant of Guillan Barré characterized by the clinical triad of ophthalmoplegia, ataxia, and areflexia [16]. However, there are other symptoms of this triad, such as changes in gait (dysplasia), astasia, and trunk ataxia. These patients were treated with immunoglobulin and acetaminophen, respectively, progressing satisfactorily [22].

Nerve alteration and SMF can occur due to an aberrant immune response to COVID-19. However, the clinical neurological spectrum in patients with COVID-19 has not yet been fully elucidated $[16,22]$.

\section{Complementary exams}

As for the laboratory findings of patients with neurological symptoms or a clinical picture of severe respiratory failure, it was observed that there are similar aspects. According to the early-onset COVID-19 diagnostic and therapeutic plan, patients may have normal leukocytes or leukopenia, regular or reduced lymphocyte count, elevated liver enzymes, LDH, muscle enzymes, 
and myoglobin. In most patients, CRP is high, and in severe cases, D-dimer is high associated with lymphopenia [20].

Such evidence shows a lower immune reaction to fight SARS-CoV-2, facilitating wide spread dissemination throughout the body. Regarding the involvement of the Peripheral Nervous System from the laboratory point of view, there was no statistical significance [1].

Through the clinical evaluation of nine children with a confirmed diagnosis of COVID-19 after hospital discharge, it was observed that one of these children evolved with mild mental symptoms and, comparatively, had elevated CRP for a more extended period than the other cases analyzed. Thus, CRP has become an essential indicator of assessment of the prognosis and severity of the disease, since it reflects the systemic inflammatory response [6,21].

Baig, et al. Believe that the search for the virus in the CSF is essential to assess the clinical evolution of the disease in patients, insisting that this biomarker can corroborate for early suspicion of neurological deficits. However, other authors consider that CSF assessment inadvertently represents only a new site of infection [3].

Despite these descriptions, clinical studies are underdevelopment, and most information is based on subjective data. As the number of patients infected with SARS-CoV-2 progressively increases in healthcare services, advanced neuroimaging tests, such as MRI and procedures such as lumbar puncture, are avoided to reduce the risks of cross-infection [1].

The brain image exam most widely used to corroborate the diagnostic hypotheses in cases with associated neurological disorders is computed tomography (CT) since it is more available in the public health system and has a lower cost [21].

However, this examination only excludes structural neurological causes, such as the presence of illnesses and brain tumors. Neurologists say that magnetic resonance imaging is more accurate to detect changes resulting from inflammatory and infectious lesions, the standard exam being another in cases of COVID-19 with nerve syndromes $[7,23]$.

\section{Neurological complications/Risk factors}

Nath $A$, et al. described the following risk factors: Advanced age, cardiac and respiratory disorders, hypertension, diabetes, in addition to kidney and liver disorders. The use of Angiotensin-Converting Enzyme Inhibitors (ACEI) in the treatment of hypertension associated with diabetes was considered a factor with a worse prognosis [5,7].

Due to the presence of ACE2 receptors in patients with SARS-CoV-2, when inhibiting ACE, there is an over expression of these receptors, leaving cells more exposed to infection by the virus [7].
Patients with underlying neurological disorders, such as Alzheimer's and Parkinson's disease, are mostly elderly patients and deserve special attention. Also, the use of immunosuppressive drugs and the need for daily care, make them more vulnerable and susceptible to infection due to greater exposure $[7,23]$.

\section{COVID-19/Neurological patient}

The spread of COVID-19 has been frightening in most centers around the world. In the USA, for example, 400,000 cases and almost 13,000 deaths have been reported to date. Thus, as the health system is unable to supply all the demand, patients are listed in priority levels to enjoy the necessary support, taking into account age, clinical condition, and prognosis, for example $[7,23]$.

Therefore, health professionals and service managers are always faced with an ethical dilemma, and neurologists are increasingly concerned about the future of neurological patients, since, in most cases, they are not considered priorities, due your primary pathology $[22,24]$.

Thus, patients with COVID-19 and associated dementia may be initially removed from ventilators due to the scarcity of resources. The neurology team and all other physicians responsible for these patients can recognize the neurological signs and symptoms early to prevent worse progression [7,24].

\section{Conclusion}

In conclusion, SARS-CoV-2 can affect the central or peripheral nervous system. Patients affected by COVID-19 associated with neurological clinical manifestations evolve with greater severity. Thus, health professionals must know the atypical signs of the disease, as patients who do not have accompanying respiratory symptoms are considered a critical hidden source of the virus's spread.

Therefore, immediate diagnostic suspicion and early identification of these patients is necessary to contain the viral transmission, avoid clinical deterioration, and reduce mortality rates. The primary purpose of this review was to assist professionals in investigating neurological changes since the onset of the condition, avoiding an increase in the transmissibility of the disease and worse clinical outcomes.

\section{Highlights}

- In a pandemic situation, symptoms such as hyposmia or hypogeusia should alert COVID-19, even when respiratory conditions are not described.

- Syncope, headache, seizures, hemiplegia, and decreased level of consciousness have been reported in positive cases for SARS-CoV-2.

- Lymphopenia, thrombocytopenia, and urea elevation correlate with the presence of symptoms in the CNS. 


\section{Acknowledgments}

The authors thank the Ph.D. in Health Sciences and Teaching and Research Manager at League Against Cancer, Profa. Dra. Amália Rêgo, for her contribution and relevance to the scientific discussion and supervision of this review, acting as an expert consultant on the bibliographic survey, analysis, and scientific advice. We also thank all the study components for their dedication and effort to build a scientifically validated quality study.

\section{Disclosure of Conflict of Interest}

There are no conflicts of interest to declare by any of the authors of this study.

\section{References}

1. Mao L, Jin H, Wang M, Hu Y, Chen S, et al. (2020). Neurologic Manifestations of Hospitalized Patients With Coronavirus Disease 2019 in Wuhan, China. JAMA Neurol 77: 683-690.

2. Steardo L, Steardo L Jr, Zorec R, Verkhratsky A (2020) Neuroinfection may contribute to pathophysiology and clinical manifestations of COVID-19. Acta Physiol (Oxf) 229: e13473.

3. Baig AM (2020) Neurological manifestations in COVID-19 caused by SARS-CoV-2. CNS Neurosci Ther 26: 499-501.

4. Chen T, Wu D, Chen H, Yan W, Yang D, et al. (2020) Clinical characteristics of 113 deceased patients with coronavirus disease 2019: Retrospectivestudy. BMJ 368: 1091.

5. Adhikari SP, Meng S, Wu YJ, Mao YP, Ye RX, et al. (2020) Epidemiology, causes, clinical manifestation and diagnosis, prevention and control of coronavirus disease (COVID-19) during the early outbreak period: A scoping review. Infect Dis Poverty 9: 29.

6. Su L, Ma X, Yu H, Zhang Z, Bian P, et al. (2020) The different clinical characteristics of corona virus disease cases between children and their families in China - the character of children with COVID-19. Emerg Microbes Infect 9: 707713.

7. Nath A (2020) Neurologic complications of coronavirus infections. Neurology 94: 809-810.

8. The World Health Organization (WHO) (2020) The First Few X (FFX) Cases and contact investigation protocol for 2019-novel coronavirus (2019-nCoV) infection. WHO/2019nCoV/FFXprotocol/2020.2 CC BY-NC-SA 3.0 IGO.

9. Zhou L, Zhang M, Wang J, Gao J (2020) Sars-Cov-2: Underestimated damage to nervous system. Travel Med Infect Dis 101642.

10. Wu Y, Xu X, Chen Z, Duan J, Hashimoto K, et al. (2020)
Nervous system involvement after infection with COVID-19 and other coronaviruses. Brain Behav Immun 87: 18-22.

11. Li YC, Bai WZ, Hashikawa T (2020) The neuroinvasive potential of SARS-CoV2 may play a role in the respiratory failure of COVID-19 patients. J Med Virol.

12. Huang C, Wang Y, Li X, Ren L, Zhao J, et al. (2020) Clinical features of patients infected with 2019 novel coronavirus in Wuhan, China. Lancet 395: 497-506.

13. Azhideh A (2020) COVID-19 Neurological Manifestations. International Clinical Neuroscience Journal 7: 54.

14. Villalba NL, Maouche $Y$, Ortiz MBA, Sosa ZC, Chahbazian JB, et al. (2020) Anosmia and Dysgeusia in the Absence of Other Respiratory Diseases: Should COVID-19 Infection Be Considered? Eur J Case Rep Intern Med 7: 001641.

15. Wang HY, Li XL, Yan ZR, Sun XP, Han J, et al. (2020) Potential neurological symptoms of COVID-19. Ther Adv Neurol Disord 13: 1756286420917830.

16. Poyiadji N, Shahin G, Noujaim D, Stone M, Patel S, et al. (2020) COVID-19-associated Acute Hemorrhagic Necrotizing Encephalopathy: CT and MRI Features. Radiology 201187.

17. Karimi N, Sharifi RA, Rouhani N (2020) Frequent Convulsive Seizures in an Adult Patient with COVID-19: A Case Report, Iran Red Crescent Med J 22: e102828.

18. Tapé C, Byrd KM, Aung S, Lonks JR, Flanigan TP, et al. (2020) COVID-19 in a Patient Presenting with Syncope and a Normal Chest X-ray. R I Med J 103: 50-51.

19. Zhao H, Shen D, Zhou H, Liu J, Chen S (2020) Guillain-Barré syndrome associated with SARS-CoV-2 infection: causality or coincidence? Lancet Neurol 19: 383-384.

20. Li CX, Wu B, Luo F, Zhang N (2020) [Clinical Study and CT Findings of a Familial Cluster of Pneumonia with Coronavirus Disease 2019 (COVID-19)]. Sichuan Da Xue Xue Bao Yi Xue Ban 51: 155-158.

21. Li B, Li X, Wang Y, Han Y, Wang Y, et al. (2020) Diagnostic value and key features of computed tomography in Coronavirus Disease 2019. Emerg Microbes Infect 9: 787-793.

22. Gutiérrez-Ortiz C, Méndez A, Rodrigo-Rey S, San Pedro-Murillo E, Bermejo-Guerrero L, et al. (2020) Miller Fisher Syndrome and polyneuritis cranialis in COVID-19. Neurology.

23. Baig AM, Khaleeq A, Ali U, Syeda H (2020) Evidence of the COVID-19 Virus Targeting the CNS: Tissue Distribution, Host-Virus Interaction, and Proposed Neurotropic Mechanisms. ACS Chem Neurosci 11: 995-998.

24. Jin H, Hong C, Chen S, Zhou Y, Wang Y, et al. (2020) Consensus for prevention and management of coronavirus disease 2019 (COVID-19) for neurologists. Stroke Vasc Neurol. 